\title{
Anesthetic management of the emergency laparotomy for a patient with multiple sclerosis -A case report-
}

\author{
Ki Hwa Lee, Jang Su Park, Sang Il Lee, Ji Yeon Kim, Kyeong Tae Kim, Won Ju Choi, and Jeong Won Kim \\ Department of Anesthesiology and Pain Medicine, Ilsan Paik Hospital, Inje University, Goyang, Korea
}

\begin{abstract}
A 33-year-old male patient with multiple sclerosis (MS) received an emergency laparotomy because of perforated appendicitis. He had been suffering from MS for 2 years and the symptoms of MS were paraplegia and urinary incontinence. Anesthesia was induced with propofol and remifentanil and maintained with nitrous oxide, sevoflurane and remifentanil. Rocuronium was used for tracheal intubation. Train of four ratio and bispectral index scale were also monitored for adequate muscle relaxation and anesthetic depth. The patient emerged from general anesthesia smoothly and was extubated without any complication. Postoperative exacerbation of MS symptoms did not appear. However, he was rehospitalized because deep vein thrombosis (DVT) occurred after discharge and he received heparinization immediately. Eventually, he was discharged after a full recovery from DVT. We report a safe anesthetic management of the patient with MS, with the use of sevoflurane and with no the aggravation of MS during postoperative period. (Korean J Anesthesiol 2010; 59: 359-362)
\end{abstract}

Key Words: Emergency laparotomy, Multiple sclerosis, Sevoflurane.

Multiple sclerosis, repeating relief and recurrence, requires unpredictable clinical course. Especially during the perioperative period, many factors can make the patients' conditions serious. Anesthesiologists must explain to the patient before the anesthesia that operation, emotional instability, anesthesia, and increased body temperature can worsen the symptoms [1]. They also must carefully pay attention to selecting the most appropriate method and drug for the anesthesia and monitoring the need for anesthesia, as well as providing a safe recovery from the anesthesia and postoperative pain control. It is of great importance to inject anxiolytics and antibiotics during the perioperative period and maintain the body temperature in the proper range in order to prevent postoperative exacerbation and recurrence [2]. In particular, a special prevention measure is necessary for multiple sclerosis patient who cannot walk, since they have a high risk of deep venous thrombosis from lying in bed for a long period of time even after the operation. No case of general anesthesia for the emergency operation of a multiple sclerosis patient has been reported yet in Korea.

Therefore, we report the case where general anesthesia was performed on a multiple sclerosis patient who needed

Received: February 11, 2010. Revised: March 4, 2010. Accepted: March 20, 2010.

Corresponding author: Jang Su Park, M.D., Department of Anesthesiology and Pain Medicine, Ilsan Paik Hospital, Inje University, 2240, Daewha-dong, Ilsanseo-gu, Goyang 411-706, Korea. Tel: 82-31-910-7160, Fax: 82-31-910-7184, E-mail: jangpark@ilsanpaik.ac.kr (c) This is an open-access article distributed under the terms of the Creative Commons Attribution Non-Commercial License (http:// creativecommons.org/licenses/by-nc/3.0/), which permits unrestricted non-commercial use, distribution, and reproduction in any medium, provided the original work is properly cited. 
emergency laparotomy using sevoflurane. Although there was no postoperative exacerbation or recurrence, deep venous thrombosis was experienced. Here, we report the case with discussion of related references.

\section{Case Report}

A male, $173 \mathrm{~cm}, 71 \mathrm{~kg}$, at the age of 33 came to the emergency room with the chief complaint of pain at the right lower abdomen that had started one day before. The patient had been diagnosed with multiple sclerosis two years previously and has been receiving long-term care by another hospital. He used to take anticonvulsants, baclofen $30 \mathrm{mg}$, and clonazepam $0.5 \mathrm{mg}$, a muscle relaxant, tizanidine hydrochloride $6 \mathrm{mg}$, and a urinary incontinence drug, oxybutynin hydrochloride $5 \mathrm{mg}$, since he had paraplegia as well as urinary incontinence at the moment of his coming to the hospital. Since perforation of gangrenous appendicitis was suspected according to emergency abdomen computed tomography, an emergency laparotomy was planned. No specific abnormal findings were observed by the blood test, electrocardiogram, or chest radiograph carried out before the operation.

Premedication was carried out by intramuscle injection of glycopyrrolate $0.2 \mathrm{mg}$ and midazolam $2 \mathrm{mg}$, and an intravenous injection of ceftriaxone $2 \mathrm{~g}$ as the preoperative antibiotic. When the patient arrived in the operating room, the electrocardiograph, noninvasive blood pressure device, capnograph, and pulse oximeter were attached, as well as a $20 \mathrm{G}$ catheter that was connected to the left radial artery. The vital signs before the anesthesia were blood pressure of $150 / 80 \mathrm{mmHg}$, heart rate of $105 \mathrm{rate} / \mathrm{min}$, oxygen saturation of $100 \%$, and body temperature of $36.7^{\circ} \mathrm{C}$. To check the depth of anesthesia during the operation, a bispectral index (BIS ${ }^{\circledR}$, Aspect Medical System, Norwood, MA, USA) was attached at the forehead before the induction. In addition, a train of four (TOF) device was connected to the right ulnar nerve to judge the muscle relaxation status. Before the anesthesia, $100 \%$ of the oxygen was put in, and $1 \%$ lidocaine $40 \mathrm{mg}$ and $1 \%$ propofol $120 \mathrm{mg}$ were injected intravenously. Then, manual ventilation was carried out with sevoflurane 2 vol\%, $\mathrm{O}_{2} 8 \mathrm{~L} / \mathrm{min}$, and remifentanil $1 \mu \mathrm{g} / \mathrm{kg}$, checking that the BIS dropped to the range of 20-30. Endotracheal intubation was performed after checking TOF reaction became zero by the intravenous injection of rocuronium $35 \mathrm{mg}$. The maintenance of anesthesia was done with sevoflurane $1-2$ vol\%, $\mathrm{N}_{2} \mathrm{O} 2 \mathrm{~L} / \mathrm{min}, \mathrm{O}_{2} 2 \mathrm{~L} / \mathrm{min}$, and remifentanil $0.5-1.5 \mu \mathrm{g} / \mathrm{kg} / \mathrm{min}$. The respiration was controlled so that the end-tidal carbon dioxide remained in the range of 30-35 mmHg. Throughout the operation, the systolic blood pressure and diastolic blood pressure were kept in the range of 120-140 mmHg and 60-70 mmHg, respectively, and the body temperature in the range of $36.5-36.7^{\circ} \mathrm{C}$ and $\mathrm{BIS}, 40-50$. The TOF ratio was 0.4 and $0.6-0.7$, respectively, after 30 minutes and 1 hour after the intravenous injection of rocuronium. The duration of anesthesia was two hours without the additional injection of rocuronium, and the operation was finished in one and half hour. After the complete skin suture, $\mathrm{N}_{2} \mathrm{O}$ and sevoflurane was stopped and ventilatory assistance was carried out with $100 \% \mathrm{O}_{2} 5 \mathrm{~L} / \mathrm{min}$, waiting for sufficient recovery of spontaneous breathing. After 2 minutes, BIS was maintained in the range of $75-85$. Then, as the TOF ratio continued to be over 0.9 , the patient could raise his head more than 5 seconds, and the consciousness was sufficiently recovered so that the patient could respond to oral directions. The endotracheal tube was extubated after confirming the sufficient recovery of spontaneous respiration and consciousness. The patient was moved to the recovery room since no specific problem occurred after the extubation. For the postoperative control of pain, fentanyl citrate $50 \mu \mathrm{g}$ was injected intravenously, and the patient no longer complained of pain. Meperidine $25 \mathrm{mg}$ was injected intravenously since tremor was observed. Cold packing was performed and $300 \mathrm{ml}$ of fluid was injected intravenously since the body temperature was raised to $38^{\circ} \mathrm{C}$. However, this did not affect the body temperature, so propacetamol hydrochloride 1 g was injected intravenously. The body temperature dropped to $37.4^{\circ} \mathrm{C} 20$ minutes after the injection.

After staying for 11 days in the ward after the operation, the patient was discharged without any complication or exacerbation of multiple sclerosis. However, 2 days after being discharged, the patient returned to the hospital with the chief complaint of right lower leg edema. By the lower extremity venous doppler ultrasound examination results, deep venous thrombosis was found from the right proximal femoral vein to the popliteal vein. Heparin was immediately injected intravenously and the patient was discharged after 7 days in better condition.

\section{Discussion}

Multiple sclerosis is a disease of random and reversible demyelination at the central nervous system [1] and is relatively rare in the Asian population [3]. It is more common in females, but in the case of male patients, as in this case report, the disorder is more serious, exacerbating and long-lasting, with a lower survival rate [4]. The symptoms include sensory abnormality, visual disturbance, weakened limbs, urination disorder, and fatigue in the afternoon [3].

Multiple sclerosis is a disease that sensitively responds to various stress factors, and before the operation is performed, it is important to inform the patient about the operation, emotional instability, anesthesia, and body temperature increase, 
since they are the exacerbation and recurrence factors of postoperative multiple sclerosis [1]. Particularly for a multiple sclerosis patient who needs an emergency operation, as in this case report, the preventive injection of an anxiolytics as premedication is necessary because emotional instability is an important exacerbation factor [2]. In addition, since the anxiety that is caused by severe postoperative pain that the patient has to suffer can worsen the symptoms of multiple sclerosis, control of the pain is one of the important roles that the anesthesiologists must play.

The medication used for the treatment of multiple sclerosis can affect the general anesthesia. Although baclofen, which was used for the patient in this case report, is an effective drug for tetany, it may cause weakening of the muscles [5]. Weakening of the muscles and reduction of muscle mass are related to an increased sensitivity to muscle relaxants [1]. Care must be taken in selecting the muscle relaxants for general anesthesia, and succinylcholine, a depolarizing muscle relaxant, should be avoided since it can cause hyperkalemia. Because there are different patient reactions to nondepolarizing muscle relaxants [6], the reaction to the muscle relaxants must be monitored by using a TOF instrument. The effect of residual muscle relaxants must be monitored with a TOF instrument because hypoventilation or atelectasis can be caused when the ventilation is not sufficiently conducted due to a delayed recovery from muscle relaxation [1].

While the spinal anesthesia is related to the exacerbation of multiple sclerosis, it has been reported that epidural anesthesia or peripheral nerve block does not cause exacerbation [6]. In the case of spinal anesthesia, the spinal cord becomes more sensitive to the neurotoxicity of local anesthetics due to demyelination, but epidural anesthesia is less dangerous than spinal anesthesia because the concentration of local anesthetics in the white matter of the spinal cord is lower than that of spinal anesthesia.

Hyperthermia during the perioperative period can be one cause of the postoperative recurrence and exacerbation of multiple sclerosis. Although, in this case report, temporary body temperature rise was observed after the operation, the temperature rise seemed to be due to body tremor, fatigue, and stress, and no sign of pneumonia, urinary tract infection, or infection at the operation site was found. The demyelinated nerve fibers of multiple sclerosis patients are extremely sensitive to an increase in body temperature. Thus, visual disturbance can be worsened by a nerve fiber conduction block or even by drinking a hot beverage [7]. Hypothalamus dysfunction has been reported to be the reason for the death of a patient who suffered periodic hyperthermia [8]. In this case report, recurrence and exacerbation of multiple sclerosis were avoided by using symptomatic treatment, such as fluid injection, cold packing, and antipyretic injection, but whether it is the central hyperthermia or not should be identified. Moreover, excessive heating must be avoided during anesthesia. The body temperature must be controlled by continuous monitoring, and antibiotics need to be injected preventively to deal with operative infection [2]. When hyperthermia is found during the operation, body temperature-dropping measures, such as operation room temperature control, cooling blanket, ice pack, and fluid treatment through the central vein, need to be taken immediately.

Deep venous thrombosis must be considered as one of the postoperative risk factors in a multiple sclerosis patient. It can occur to a patient who has to stay in bed for a long period of time due to leg paralysis. Since the probability of deep venous thrombosis for patients of developed multiple sclerosis is $43.9 \%$, appropriate preventive measures are recommended [9]. The preventive measures include pharmacotherapy using anticoagulants, wearing compression stockings, intermittent external pneumatic compression, and early ambulation after operation [10]. In this case report, appropriate preventive measures could not be taken since it was an emergency operation, and early postoperative ambulation was also impossible due to the limb paralysis. Thus, the patient was included in the high risk group of deep venous thrombosis, which was eventually found 2 days after the patient's discharge. The patient was able to be discharged again without any complication.

There is a case report where general anesthesia for a multiple sclerosis patient was safely carried out using sevoflurane, which has a low blood/gas distribution coefficient. The report says that sevoflurane is not related to postoperative exacerbation or febrile event [11]. There has been no case report of anesthesia using desflurane. No publication is available regarding which type of intravenous and inhalation anesthetics is best for the patients.

Multiple sclerosis is a chronic neurological disease that causes disability, gradually making the patients lose movement ability and eventually causing limbs paralysis. The most important aspect of anesthetic management for a multiple sclerosis patient is noting that the disease's course cannot be anticipated and the postoperative or postpartum exacerbation of the preexisting disorders must be prevented as much as possible. Further, since the hemodynamic signs can severely fluctuate due to autonomic nervous dysfunction during the operation, invasive arterial pressure measurement is required. Also, postoperative neurological examination must be carried out immediately. In this case report, the postoperative neurological examination was carried out by consultation with the department of neurology, and no significant abnormality was found. Preventive anxiolytics medication, body temperature control, 
and antibiotic injection during the perioperative period can prevent the recurrence and exacerbation of multiple sclerosis [2].

Regardless of the anesthesia method used in the elective operation of a multiple sclerosis patients, the operation must be delayed if the symptoms are being exacerbated [12]. The anesthesia plan must be made after sufficiently considering the type of the disease, severity, and neurological state of the patient before the operation [1]. However, in the case of an emergency operation where delay is impossible, as in this case report, perioperative anesthesia management is required to prevent exacerbation and recurrence of the symptoms.

\section{References}

1. Dorotta IR, Schubert A. Multiple sclerosis and anesthetic implications. Curr Opin Anaesthesiol 2002; 15: 365-70.

2. Dickerman RD, Schneider SJ, Stevens QE, Matarese NM, Decker RE. Prophylaxis to avert exacerbation/relapse of multiple sclerosis in affected patients undergoing surgery: surgical observations and recommendations. J Neurosurg Sci 2004; 48: 135-7.

3. Noseworthy JH, Lucchinetti C, Rodriguez M, Weinshenker BG. Multiple sclerosis. N Engl J Med 2000; 343: 938-52.

4. Kantarci OH, Weinshenker BG. Natural history of multiple sclerosis.
Neurol Clin 2005; 23: 17-38.

5. Mitchell G. Update on multiple sclerosis therapy. Med Clin North Am 1993; 77: 231-49.

6. Pasternak JJ, Lanier WL Jr. Spinal cord disorders. In: Stoelting's Anesthesia and Co-existing Disease. 5th ed. Edited by Hines RL, Marschall KE: New York, Churchill Livingstone. 2008, pp 230-1.

7. Matthews WB, Read DJ, Pountney E. Effect of raising body temperature on visual and somatosensory evoked potentials in patients with multiple sclerosis. J Neurol Neurosurg Psychiatry 1979; 42: 250-5.

8. Martinez-Rodriguez JE, Munteis E, Roquer J. Periodic hyperthermia and abnormal circadian temperature rhythm in a patient with multiple sclerosis. Mult Scler 2006; 12: 515-7.

9. Arpaia G, Bavera PM, Caputo D, Mendozzi L, Cavarretta R, Agus $\mathrm{GB}$, et al. Risk of deep venous thrombosis (DVT) in bedridden or wheelchair-bound multiple sclerosis patients: a prospective study. Thromb Res 2010; 125: 315-7.

10. Gonzalez M. Vascular disease. In: Stoelting's Anesthesia and Coexisting Disease. 5th ed. Edited by Hines RL, Marschall KE: New York, Churchill Livingstone. 2008, pp 156-7.

11. Inoue S, Furuya H. Sevoflurane is safe for anesthetic management in patients with multiple sclerosis. Acta Anaesthesiol Taiwan 2006; 44: 187-9.

12. Morgan GE, Mikhail MS, Murray MJ. Clinical anesthesiology. 4th ed. New York, The McGraw-Hill Companies. 2006, pp 652-3. 